\title{
tic\&société
}

Vol. 14, $\mathbf{N}^{\circ}$ 1-2 | 1er semestre 2020 - 2ème semestre 2020

Mutations numériques de la musique : des

contradictions à analyser

\section{Vues vérifiées, écoute évacuée : la valorisation publicitaire de la musique sur YouTube}

Verified hits, listening optional: the publicity value of music on YouTube

Visión verficada, escucha marginada : la valorización publicitaria de la música

en youtube

\section{Guillaume HEUGUET}

\section{CpenEdition}

Journals

Édition électronique

URL : http://journals.openedition.org/ticetsociete/4782

DOI : $10.4000 /$ ticetsociete.4782

Éditeur

Association ARTIC

Édition imprimée

Pagination : 95-129

Référence électronique

Guillaume HEUGUET, «Vues vérifiées, écoute évacuée : la valorisation publicitaire de la musique sur YouTube », tic\&société [En ligne], Vol. 14, № 1-2। 1er semestre 2020 - 2ème semestre 2020, mis en ligne le 11 novembre 2020, consulté le 24 février 2021. URL : http://journals.openedition.org/ ticetsociete/4782 ; DOI : https://doi.org/10.4000/ticetsociete.4782 
tic\&société - 14(1-2), 2020

\title{
Vues vérifiées, écoute évacuée : la valorisation publicitaire de la musique sur YouTube
}

\begin{abstract}
Guillaume HEUGUET
Guillaume Heuguet est docteur en sciences de l'information et de la communication au GRIPIC (Celsa-Paris Sorbonne) et postdoctorant à Sorbonne Université. II est rédacteur en chef de la revue Audimat, qui relie critique musicale et critique sociale. quillaume@inparadisum.net
\end{abstract}


Vue vérifiées, écoute évacuée :

la valorisation publicitaire de la musique sur YouTube

\section{Vues vérifiées, écoute évacuée : la valorisation publicitaire de la musique sur YouTube}

Résumé: Depuis 2013, YouTube est reconnu comme la " première destination » des auditeurs de musique en France. Fondée par trois anciens employés de PayPal en 2005 comme l'éditrice d'un site de stockage et de publication de vidéos, l'entreprise a peu à peu développé un système technique et un modèle d'affaires qui entendent "monétiser » la musique publiée sur le site en articulant l'écoute "à la demande » avec un système de régie publicitaire. S'appuyant notamment sur une veille sur différents espaces de youtube.com, le blogue YouTube et l'étude de brevets, cet article s'intéresse aux médiations techniques, sémiotiques et symboliques sur lesquelles repose la valorisation marchande de la musique dans un dispositif médiatique généraliste d'abord conçu pour la vidéo. II analyse l'hétérogénéité des calculs et des signes liés à une mesure d'audience spécifique, la "vue ». II montre ensuite son statut d'étalon contesté parmi les acteurs du marché de la musique. II interroge enfin les aspects anthropologiques des normes de l'économie et de la consommation des médias qui structurent l'acceptabilité d'une telle activité de mesure. Entre la prétention de YouTube à se situer au plus près des pratiques musicales des internautes pour les attirer et séduire les annonceurs et un mode de calcul conçu indépendamment de tout rapport spécifique à la musique, la médiation des valeurs de la musique sur YouTube apparaît comme un paradoxe.

Mots-clés: musique, streaming, YouTube, économie de l'attention, publicité, innovation, théorie critique.

\section{Verified hits, listening optional: the publicity value of music on YouTube}

Abstract: Since 2013, YouTube has been considered the "top destination" for music listening in France. Launched by three 
employees from PayPal in 2005 as editor of a website for hosting and publishing videos, the company has developed a technical system and a business model that monetises published music on the website by linking "on demand" listening with an advertising control system. Based on a media survey including YouTube.com, the YouTube blog and the study of patents, this paper uses a socio-semiotic approach to analyse technical, semiotic and symbolic mediations which support the commercial valuation of music in a generalist media apparatus initially designed for video. It first analyses how a range of technosemiotic objects convert a multiplicity of signs into financial value. It then shows how reference to a specific audience measurement, the "view", is a disputed standard among music market agents. Finally, it explores the anthropological aspects of media economics and consumption behaviour that support the acceptance of this measure of activity. Between Youtube's claims to best represent web users' musical interests and practices to attract them and seduce brands, and a technical system designed to calculate the monetary value of music without reference to musical form or practice, mediating the value of music on YouTube appears contradictory.

Keywords: music, streaming, YouTube, attention economy, advertising, innovation, critical theory.

\section{Visión verficada, escucha marginada : la valorización publicitaria de la música en YouTube}

Resumen: Desde 2013, YouTube es considerado como el "primer destino" para escuchar música en Francia. Lanzada por tres empleados de PayPal, en 2005, como editor de un sitio web para hospedar y publicar videos, la empresa ha desarrollado un sistema técnico y un modelo de negocio que busca "monetizar" la música publicada en el sitio web que se "bajo demanda" y que se financia con publicidad. El artículo estudia las mediaciones técnicas, semióticas y simbólicas sobre las que reposa la 
Vue vérifiées, écoute évacuée :

la valorisation publicitaire de la musique sur YouTube

valorización mercantil de la música en un dispositivo mediático generalista, especialmente concebido desde sus inicios para el video. Para ello se estudian diferentes espacios de YouTube.com, el blog de YouTube y las patentes. Previamente analiza la heterogeneidad de cálculos y de signos relacionados con la medida de una audiencia específica, la "vista"-; para con posterioridad mostrar cómo la totalidad de actores de la industria musical ponen en cuestión el estándar de medición. Posteriormente se interesa por los aspectos antropológicos de las normas económicas y del consumo de medios de comunicación que estructuran la aceptabilidad de un determinado patrón de medida.

Palabras claves: musica transmisión, YouTube, economía de la atención, publicidad, innovación, teoría crítica. 
Lancée en 2005 par trois anciens employés du site de paiement en ligne PayPal, YouTube Inc. a commencé par éditer un site et une application offrant aux internautes d'héberger et de publier leurs vidéos, en particulier des "blogues vidéos » (vlogs), des sketchs et des montages audiovisuels (Hillrichs 2005). Comme l'ont révélé les e-mails des fondateurs lors d'un procès l'opposant à VIACOM ${ }^{1}$, l'entreprise a décidé de laisser publier les vidéos incorporant des œuvres musicales soumises au droit d'auteur, en même temps qu'elle commençait à inviter certains artistes et labels indépendants à déposer et à promouvoir leurs propres clips musicaux.

En 2006, Google - rebaptisée en 2015 Alphabet Inc. - a acquis YouTube et versé plusieurs millions de dollars aux principales majors du disque (Mulligan 2015). Si les détails de cet accord ne sont pas connus, il semble avoir permis d'étendre une période de tolérance de la part de celles-ci, accordant à YouTube une place privilégiée dans les pratiques d'écoute de musique en ligne sous forme de flux (streaming). Les premières publicités sont introduites en 2007. En 2009, YouTube développe Vevo, un modèle éditorial et économique de " chaînes vidéos » rémunérées par la publicité pour diffuser spécifiquement le catalogue de trois des quatre majors (Mclntosh 2016), ainsi qu'un système de détection des œuvres musicales soumise au droit d'auteur dans les vidéos dites "générées par les utilisateurs" (Content ID) afin de les « monétiser » à leur tour (Eriksson, 2019 ; Heuguet, 2019).

En 2013, l'entreprise était ainsi devenue la «première destination pour l'écoute de musique en ligne » en France ${ }^{2}$. Profitant des moyens de Google, parmi d'autres facteurs comme l'intégration de son lecteur média aux pages MySpace et la fermeture provisoire de ce « réseau social » de premier plan ${ }^{3}$

1 Electronic Frontier Foundation. (s. d.). VIACOM v. YOUTUBE. Récupéré de https://www.eff.org/cases/viacom-v-youtube

${ }^{2}$ Carpentier, L. (2013, 31 octobre). YouTube, machine à tubes. LeMonde.fr. Récupéré de https://www.lemonde.fr/culture/article/2013/10/31/youtube-machine-atubes 3506517 3246.html

3 Keegan, V. (2007, 8 février). Will MySpace ever lose its monopoly?. The Guardian. Récupéré 
Vue vérifiées, écoute évacuée :

la valorisation publicitaire de la musique sur YouTube

-, YouTube a joué un rôle important dans les pratiques de partage et d'écoute de musique en ligne. L'entreprise a participé à ré-encastrer ces pratiques dans un marché hybride, situé à l'intersection des processus de valorisation de la musique, des technologies et des médias (Wade Morris 2015).

Pourtant, la reconstruction de transactions régulées autour de l'échange de la musique en ligne n'allait pas de soi. Jean-Samuel Beuscart (2006) a décrit les difficultés rencontrées par les entreprises françaises qui expérimentaient des modèles techniques, éditoriaux et économiques pour capitaliser sur ces pratiques. II souligne la logique d'expérimentations « à ciel ouvert " et le mouvement de balancier qui s'opère entre les intermédiaires émergents et les maisons de disques:

Sur le marché de la musique, les innovations de diffusion de musique en ligne se développent dans un premier temps hors de tout cadre réglementaire, ou en fonction d'anticipations très incertaines sur les règles pertinentes du marché. Elles débordent largement les règles - du droit d'auteur en particulier - qui construisaient jusque-là l'équilibre de la diffusion musicale. Leur développement génère en retour la protestation des acteurs de l'industrie musicale, qui identifient des effets négatifs de la diffusion numérique sur leur activité, et travaillent à la construction de nouvelles règles afin de corriger ces externalités (p. 24).

Dans ce processus d'allers-retours, les industriels des technologies et des médias comme Google, les maisons de disques et les pouvoirs publics auront néanmoins fini par s'entendre, au moins provisoirement, pour « déléguer la charge de l'accord et du respect des règles à des dispositifs techniques qui cadrent les échanges et la concurrence " (Beuscart, 2006, p. 21): des formats et des outils de contrôle de la copie, de filtrage des publications, de mesures d'audience ou d'enchères publicitaires servent ainsi d'appui pour organiser des consensus et soutenir la réorganisation des " chaînes de valeur ".

Ces dispositifs sont en apparence doublement " techniques " : ils ont un caractère essentiellement opératoire et ils supposent un certain degré d'expertise. Ils n'en ont pas moins une portée culturelle et sociale relativement importante, puisque c'est en partie à travers eux que les valeurs de la musique se trouvent aujourd'hui ré-énoncées et recalculées. 
Sur YouTube, la reconstruction de la valeur marchande de la musique passe en effet par les multiples références aux " nombres de vues » et par les outils de mesure de l'audience des vidéos, des œuvres musicales et des publicités. C'est cet ensemble que cet article se propose d'analyser, pour étudier comment la (re)construction de la valeur commerciale de la musique en ligne incarne, réciproquement, une certaine idée des formes et de l'écoute musicale.

Dans les mesures d'audience, les catégories sémiotiques mobilisées (la « vue », mais aussi « l'intérêt »), tout comme les différentes unités de calcul retenues selon les espaces et les moments (décompte de clics, de durée, etc.) engagent une définition des usages et des valeurs de la musique. Cette médiatisation de la valeur de la musique ne transparaît pas toujours en tant que telle dans les discours des acteurs, y compris en cas de controverses. C'est la nécessaire prise en compte de ces aspects normatifs discrets puisqu'inscrits dans l'infrastructure des médias et de la culture (éditorialisation des procédures techniques sous-jacentes dans les pages web, intégrations de catégories sémiotiques et culturelles dans les modes de calculs) que nous avons caractérisée ailleurs, à la suite des travaux de Jonathan Sterne (2018), comme relevant d'une "micropolitique des formats" (Heuguet 2019), une perspective qui participe des recherches en sciences de l'information et de la communication soucieuses d'articuler les médiations socioéconomiques et techno-émiotiques pour analyser l'industrialisation de la communication (Jeanneret, 2014 ; Mœglin, 2015) - dont la réputée " plateformisation » (Bullich, 2018; Rebillard et Smyranios, 2019). Elle s'inscrit également dans une série de recherches en sciences de l'information et de la communication (SIC) sur les différentes médiations marchandes de la musique en ligne et leur portée culturelle (Bullich, 2015 ; Debruyne, 2015 ; Gras, 2014 ; Perticoz et Matthews, 2012).

Comprendre la médiatisation de la valeur de la musique par l'étude des formats permet plus particulièrement de rapprocher les apports de la sociologie économique, traditionnellement attentive au rôle des techniques dans la formation des marchés, et l'analyse sociosémiotique des médias, en particulier des « médias informatisés » (Souchier et Jeanneret, 2009).

Muniesa, Millo et Callon (2007) se sont ainsi intéressés aux 
Vue vérifiées, écoute évacuée :

la valorisation publicitaire de la musique sur YouTube

« instruments de marché » (markets devices), un concept qui recouvre les "assemblages matériels et discursifs qui interviennent dans la construction des marchés ${ }^{4}$ » (p. 2) et sans lesquels ils ne peuvent exister. Ces instruments de marché assurent la coordination d'une multitude d'acteurs, la commensurabilité des produits et la régularité des échanges. Leur éventail s'étend " des techniques d'analyse aux modèles de prix, des paramètres d'achat aux outils de marchandisage, des protocoles commerciaux aux indicateurs agrégés ${ }^{5}$ » (Muniesa, Millo et Callon, 2007, p. 2), et leur observation ouvre à une perspective pragmatique sur le processus de construction des marchés et de valorisation d'entités comme les enregistrements sonores. Les mesures d'audience dans les médias en constituent un cas d'école (Méadel, 2010), et ses perspectives peuvent être prolongées en direction d'une étude des usages de la référence à la « vue » et de ses appareillages.

Si l'analyse concrète des mesures d'audience implique de traiter de discours et d'outils, elle requiert aussi de prendre un compte, plus largement, un ensemble relativement dense de signes et de pratiques d'interprétation. En l'occurrence, les "vues " ont aussi le statut de « traces médiatisées " (Jeanneret 2019) : la référence aux "vues » vise à rendre crédible et à orienter la saisie des usages des médias en ligne. Cette approche invite à observer la polyphonie des signes tels qu'ils se présentent dans leur situation d'énonciation, ainsi que l'hétérogénéité des lectures dont ils peuvent faire l'objet. Nous verrons que les multiples occurrences de la « vue » lui confèrent des significations ambigües qui ont des conséquences du point de vue de la valorisation des pratiques musicales.

Pour interroger la (re)construction de la valeur de la musique sur YouTube, notre analyse s'appuie principalement sur une observation sémiologique et une veille médiatique au long cours (2012-2018), complétée par des mises à jour plus récentes, ainsi que sur la presse spécialisée dans les technologies et l'économie de la musique (notamment les magazines en ligne Techcrunch, The Verge, Music Ally) menée dans le cadre d'une thèse de doctorat (Heuguet 2018). Nous nous appuierons plus

\footnotetext{
4 Traduction libre de : «the material and discursive assemblages that intervene in the construction of markets ".

5 Traduction libre de : "From analytical techniques to pricing models, from purchase settings to merchandising tools, from trading protocols to aggregate indicators ».
} 
particulièrement sur les matériaux documentaires suivants ${ }^{6}$ :

1) l'éditorialisation de la mesure d'audience à travers les signes des "vues " dans différents espaces ou outils labellisés du domaine youtube.com et l'outil Google AdWords, devenu Google Ads ;

2) un brevet publié par Google en 2012 concernant le système de recommandation des vidéos, qui permet de comprendre le lien entre les « vues », les « mots-clés » publicitaires et l'enregistrement d'indices de l'activité des internautes ;

3) une série de pages d'explicitation des différentes mesures d'audience proposées par YouTube, en particulier Impact Metrics, qui présente ce qui est compté comme "vue » au sein de la YouTube Creator Academy, sous-domaine de youtube.com à vocation d'accompagnement des internautes vers la valorisation de leur chaîne, et une aide à la lecture de l'indicateur "Watch Time» (durée de visionnage) dans la fiche Watch Time Report sur YouTube Help (non daté) ;

4) les pages qui communiquent sur la fiabilité de la mesure auprès d'entreprises spécialisées dans la gestion de statistiques ou la création de campagnes publicitaires (YouTube Creator Services Directory et YouTube True View sur Thinkwithgoogle, le billet Making ads more interesting sur le Google blog), ainsi que des extraits de presse en ligne spécialisée dans les technologies rendant compte des tentatives de triche et des critiques de la mesure (Techcrunch, CNET).

D'abord, nous nous intéresserons à la logique d'ensemble de la « vue » comme mesure d'audience publicitaire permettant de synthétiser des signes hétérogènes, d'articuler des espaces techniques et discursifs, de produire des équivalences. Nous

\footnotetext{
6 L'étude de dispositifs médiatiques très dynamiques comme YouTube, et en l'occurrence d'un phénomène polymorphe tel que la construction d'une mesure d'audience, appelle un style de recherche qu'Yves Jeanneret (2014) décrit comme " ambulatoire ». La structuration de ces dispositifs comme leurs effets dépendent de multiples projets, d'espaces, de textes et d'usages multiples, dont il importe de suivre les indices " au fil de l'eau », liant l'enquête sémiologique et documentaire à l'ethnographie de la médiatisation partielle d'une culture professionnelle en partie intersectorielle. Ce n'est donc que dans un second temps, rétrospectif, qu'il est possible d'en baliser plus ou moins les contours.
} 
Vue vérifiées, écoute évacuée :

la valorisation publicitaire de la musique sur YouTube

verrons ensuite que cette dynamique d'homogénéisation n'est pas parachevée puisque la référence à la " vue » fait l'objet de différents types de critiques. Enfin, nous nous intéresserons plus précisément à l'articulation entre décompte de consommation des vidéos et calcul de l'audience des œuvres musicales qu'elles incorporent. Nous ferons ainsi apparaître la fragilité de la référence à la « vue » pour calculer la valeur économique de la musique et les contradictions de l'assemblage sémiotechnique qui la soutient.

\section{La convertibilité des signes et des valeurs}

Avec l'introduction de la publicité sur YouTube à partir de 2006, les compteurs de "vues " associés aux vidéos passent d'un critère de classement documentaire à un indicateur marchand, susceptible de déterminer des valeurs et des flux de rémunération. Toutes les formes de vidéos et de musique, toutes les valeurs de "créativité » et de "popularité » musicales pourraient alors paraître se dissoudre dans l'indifférence du chiffre. En réalité, les calculs des "vues" recouvrent un processus dense de saisie et de conversion entre de multiples activités, inscriptions et symboles. Ils font converger la fantasmagorie du chiffre et du succès avec les cultures professionnelles de la télévision, du marketing et de l'informatique. En tirant le fil de la "vue », on obtient ainsi une série étendue de catégories, de valeurs et de formats, qui participent de la densité qu'a gagnée cette idée de "vue » comme trace de popularité et de succès, à la fois incontournable et contestée.

\subsection{Un opérateur d'équivalence marchand}

La "vue " est un objet composite et communicationnel par excellence : ni tout à fait signe, concept ou pratique, elle est un peu de tout cela à la fois, elle " fait tenir » les relations entre des documents, des acteurs et des idées, dans une série de situations. Cette multidimensionnalité est vraie de toute mesure d'audience, et c'est en même temps ce qu'elle doit nier en opérant un "alignement de tout un ensemble d'objets et d'acteurs » (Méadel, 2010). Les "vues » sont en effet reliées à une variété de pratiques sociales: écouter de la musique, visionner une vidéo, mesurer la composition d'une audience, 
publier une publicité et établir son coût de diffusion, comparer les courbes statistiques situées dans le temps et dans l'espace avec des actions publicitaires ciblées, ou encore identifier les sites externes qui ont mené vers la vidéo. La référence à la « vue » coordonne ces pratiques au double niveau des inscriptions (les " données ») et des symboles (à commencer par les chiffres), de manière à organiser l'infrastructure d'un marché ${ }^{7}$. Son statut est soutenu par des articles de presse, des publicités, des communiqués de presse des maisons de disque, des conversations ordinaires. Si la "vue » est l'objet de tous les regards, elle institue en retour une variété de relations : entre les internautes (dans leur identité "profilée ») et leurs pratiques; entre les annonceurs (via leurs comptes) et les résultats de leurs investissements; et entre les musiciens et leurs publics. En créant des chaînes d'équivalence entre une série d'activités, elle participe à instaurer le marché ou à en soutenir la définition : elle sert de repère dans des situations concrètes d'échange (comme les écrans où il s'agit de payer pour diffuser un message publicitaire) comme dans la structure abstraite qui fait du marché un ensemble de pratiques régulières d'échange de valeurs et une structure de concurrence ${ }^{8}$.

\subsection{La mise en relation des pratiques culturelles et des stratégies publicitaires}

Les « vues " ont en effet vocation à transformer des pratiques en signes et des signes en vecteurs de transactions sur le marché de l'espace publicitaire. Elles ne sont pas les seuls indicateurs de l'activité de l'internaute : les « pouces » haut/bas

7 II faut évacuer ici toute opposition parasitaire entre «infrastructure » et "superstructure »: les techniques de la logistique et du calcul sont tissées de représentations (force du formulaire, de la liste et du tableau, idées comme la consistance, la fiabilité ou l'efficacité), mais leur rôle est de soutenir, de manière systématique, une organisation de la communication. L'« infra » ne désigne pas non plus le secret absolu ou l'oubli dans l'ordinaire des pratiques. Le déficit de culture technique et de connaissances sur les dimensions situées des pratiques économiques fait autant pour la « naturalisation » des infrastructures de marché que le " secret des algorithmes ». Sur la dimension matérielle et l'ethnographie des marchés, voir Callon, Licoppe et Muniesa, 2003.

${ }^{8}$ L'anthropologie économique de Polanyi (2011) attire notre attention sur le fait que, dans certaines sociétés, la monnaie ne correspond pas forcément à un unique objet ou type d'objet et qu'elle sert le plus souvent une pluralité de fonctions, en particulier : « moyen de paiement ; étalon de valeur ou monnaie de compte ; réserve de valeur ; et enfin moyen d'échange " (p. 163). Polanyi remarque en outre que la monnaie comporte une dimension métrologique, dans la mesure où elle renvoie à des objets physiques. 
Vue vérifiées, écoute évacuée :

la valorisation publicitaire de la musique sur YouTube

situés sous les vidéos en font également partie. Aux côtés des commentaires, ils s'inscrivent dans la panoplie d'une culture médiatique de l'évaluation (Bouchard et al., 2015). Les chiffres de vues représentent néanmoins l'un des principaux moyens de réduire la multiplicité des médiations culturelles et documentaires vers le domaine du calcul des valeurs d'échange.

Les chiffres de vues associés à une vidéo passent ainsi par une série de rééditorialisations, associés à d'autres chiffres qui valent comme indicateurs complémentaires selon la posture de lecture et d'utilisation impliquée. Ils deviennent le signe d'une popularité-vélocité sous une vidéo particulière en page d'accueil, valent pour l'étude d'une moyenne de captation de spectateurs et de leur conversion en abonnés dans YouTube Insights (devenu YouTube Analytics ${ }^{9}$ ), comme le repère d'une tendance de popularité hebdomadaire considérée en relatif sur les classements de YouTube for Artists, ou encore une unité de change - sous la forme d'un ratio vue/somme investie - dans Google Ads (figures 1 à 4).

\section{$1,1 \mathrm{k}$ vues $\cdot$ il y a 2 heures}

Figure 1. Le compte des vues tel qu'il est sémiotisé sous une vignette de vidéo sur youtube.com

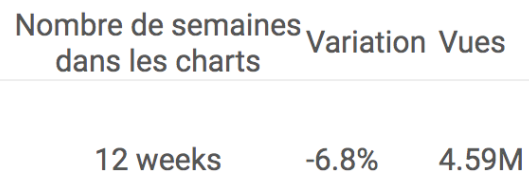

Figure 2. Le compte des vues tel qu'il est sémiotisé dans l'outil YouTube Analytics

\footnotetext{
9 II s'agit des outils de suivi des statistiques de visionnage des vidéos dans les espaces réservés aux utilisateurs inscrits.
} 


\section{Guillaume HEUGUET}

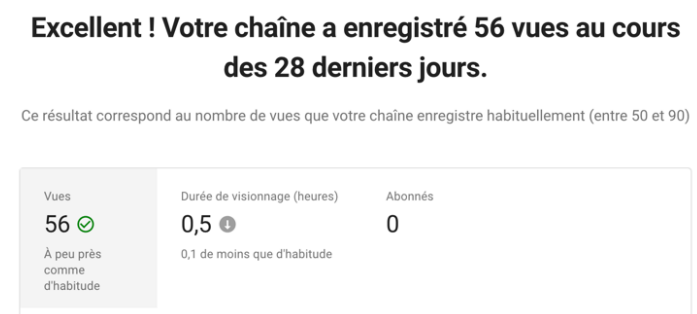

Figure 3. Le compte des vues tel qu'il est sémiotisé dans l'outil YouTube for Artists, onglet « Charts »

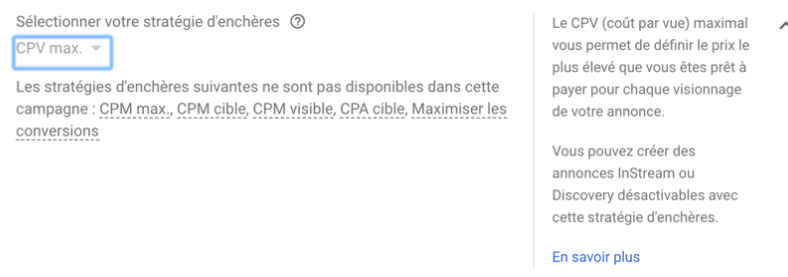

Figure 4. Le compte des vues tel qu'il est sémiotisé dans l'outil Google Ads

Tous ces dispositifs donnent une lisibilité et une intelligibilité aux chiffres de vues, qu'il s'agit chaque fois de réinterpréter différemment, même si, hormis dans AdWords, ils sont toujours reliés à une périodicité donnée, afin de lire une tendance statistique, de surveiller des évolutions et d'en tirer des stratégies.

La « vue » se révèle un opérateur d'équivalence puissant, qui permet de rassembler dans une même unité un grand nombre d'entités ${ }^{10}$. Ce traitement ne s'effectue pas à l'échelle d'un unique média compris comme support, mais de groupes de vidéos, indexées sur des "thèmes » qui correspondent euxmêmes à des mots-clés. AdWords ne propose donc pas l'association du format publicitaire à un programme ou à un créneau horaire particulier, mais à un " lot » de vidéos reliées à des mots-clés et à des situations d'utilisation projetées. Les postures et les activités mises en avant par l'outil sont directement liées à des formats d'affichage. À la liaison des

10 En économie, la « puissance » se définit comme " puissance de calcul », c'est-àdire par le plus ou moins grand nombre d'entités traitables par un opérateur d'équivalence. Cf. (Callon et Muniesa, 2003) 
Vue vérifiées, écoute évacuée :

la valorisation publicitaire de la musique sur YouTube

investissements publicitaires à des mots-clés ou des thèmes s'ajoute le choix de formats d'exposition introduits en 2015. Parmi les différents formats disponibles à partir des enchères de Google AdWords (devenu Google Ads), on trouve notamment InStream, une offre qui désigne au départ les vidéos pre-rol/ ${ }^{11}$, désormais disponibles en mid-roll et post-roll et aujourd'hui disponible sous le nom True View In-Stream Ads (figure 5).

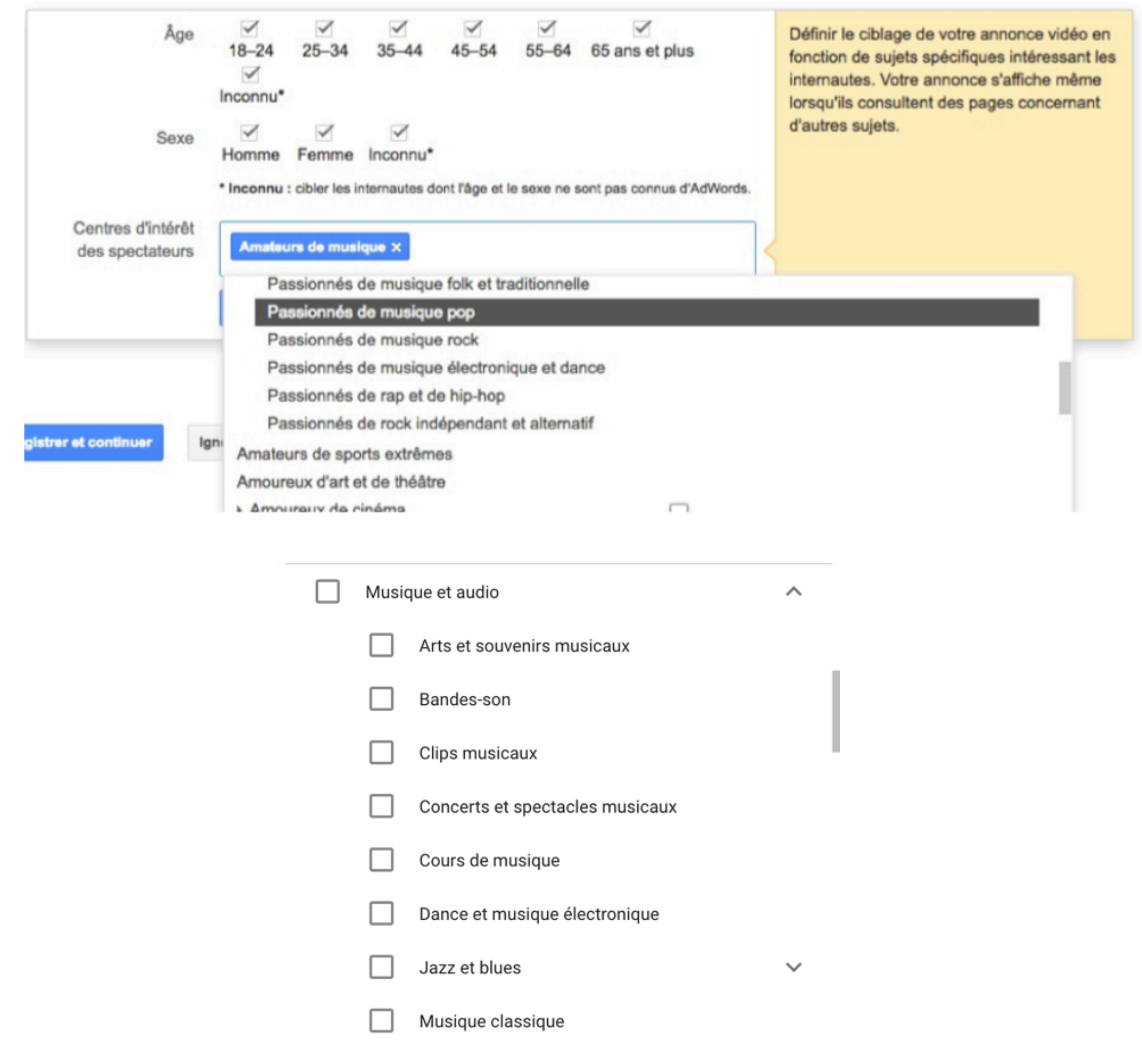

Figure 5. Les « centres d'intérêt » associés à des promesses de " vues » par lot, dans l'outil de campagne publicitaire

Google Adwords (2015)

Ils sont devenus des " thèmes » dans Google Ads (en 2020) et leur liste s'est étoffée. Le lien des nouveaux items avec les formats de vidéos et les genres musicaux indexés par YouTube est manifeste.

11 Le nom et le regroupement de ces formats ont évolué depuis 2015, mais les emplacements possibles sont restés du même ordre. 


\section{Guillaume HEUGUET}

L'annonceur qui mise sur des mots-clés peut les sélectionner parmi des suggestions, pour cibler des recherches précises ou des vidéos relativement spécifiques ou, au contraire, passer par des thèmes préformatés qui sont censés rendre compte des " centres d'intérêt» des internautes. Leur liste, qui peut ressembler à la liste des thématiques d'un portail, fonctionne en même temps comme un dictionnaire de l'imaginaire culturel et social visé par les annonceurs, définissant le point où la sémantique des modes de vie rencontre le marketing de l'offre (avec des catégories comme " passionnés de la vie nocturne », « passionnés de musique rock ") $)^{12}$.

\subsection{L'indice d'un agrégat d' " intérêts » reconstruits}

Les mots-clés, et plus encore les thèmes, fonctionnent ici comme des repères documentaires pour une multiplicité de pages et de vidéos. Le brevet du « système de recommandation de vidéos » publié en 2012 par Google ${ }^{13}$, même si celui-ci a depuis été largement retravaillé dans sa structuration ${ }^{14}$, offre un aperçu des logiques qui président à la constitution de ce type d'agrégats. Les gestes de l'internaute (saisie, interprétation, visionnage, écoute, lecture, clic) y sont notamment transformés en signaux manipulables (inputs), en tracé dans des bases de données visuelles de différents ordres et en opérations dans le code informatique. Le ciblage des internautes et l'assignation des publicités à leurs "profils » s'appuient sur ce système : il donne à voir comment les activités de l'internaute y deviennent des values labels $s^{15}$ qui permettent aussi bien d'apparier les publicités à des vidéos que de générer des prescriptions. Les

\footnotetext{
12 Les « centres d'intérêt " multipliés, précisés et rebaptisés en tant que " thèmes » apparaissent, pour une part, plus directement liés à des types de vidéos musicales regardées sur YouTube, preuve, s'il en fallait, du potentiel spécifique du site comme attracteur pour les stratégies des annonceurs, au-delà de la logique globale de Google d'annonces par mots-clés.

13 Video-related recommendations using link structure (US8145679B1). Voir : https://patents.google.com/patent/US8145679/en?oq=US+8\%2c145\%2c679+B1.+U S12966747.

${ }^{14}$ Voir le livre blanc: Covington, P., Adams, J. et Sagin, E. (2016). Deep Neural Networks for YouTube Recommendations. Récupéré de https://static.googleusercontent.com/media/research.google.com/fr//pubs/archive/455 30.pdf

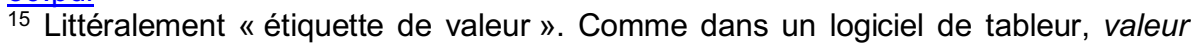
désigne une inscription qui peut avoir une dimension sémantique ou chiffrée, et que l'on peut traiter et manipuler, ce qu'un vocabulaire plus courant exprime comme une « donnée ».
} 
Vue vérifiées, écoute évacuée :

la valorisation publicitaire de la musique sur YouTube

phénomènes traqués pour devenir des inputs dans la détermination de recommandations apparaissent très variés: " contenu du profil de l'utilisateur », " contenu du log d'activité de l'utilisateur", "historique de contenu vidéo "; et ils se subdivisent encore : en "amitiés », en " appartenance à des groupes », en " commentaires postés », en « web logs postés », en «messagerie électronique », en «informations démographiques ». Les logiques décrites par ces graphes, comme les différentes entités et les différents algorithmes qu'ils modélisent, ne cessent évidemment d'évoluer dans leur logique comme dans leur contenu. Le brevet indique néanmoins un esprit général : il s'agit de croiser les activités d'un internaute avec celles d'autres utilisateurs ayant regardé au moins une partie des mêmes vidéos, ainsi que des "groupes d'utilisateurs ». Dans ce processus, une partie au moins des étiquettes censées produire des recommandations " personnelles » apparaissent en fait comme des catégories prévisibles : des "genres de vidéos », comme "comédie » ou " musique ».

Cette lecture en surface du brevet fournit un nouveau regard sur la " mise en circuit » des pratiques, des formes (à partir des genres), des vues et des vidéos publicitaires. Une série de médiations doit permettre de convertir des signes et des objets socialement et sémiotiquement " épais " pour en faire autant de relais dans un circuit $^{16}$. Différentes réalités ou réalités apparentes, comme des gestes, des signes, des vidéos, des données chiffrées, des mots-clés, sont reliées entre elles et traitées selon des principes de mise en équivalence, et une partie importante de ces processus est assumée par le « système de recommandation ». La calculabilité des choses passe ainsi par toute une série d'étapes qui leur confère au passage leur épaisseur sociale et apparemment objective. C'est au fil de ce processus que se constitue, se réinvente et se distribue la valeur marchande des pratiques et des vidéos.

\footnotetext{
16 II s'agit de la conversion d'une information 2 (information socialement interprétable) en une information 1 (information convertible en traitement automatique à fondement mathématique, l'exécution d'un programme) (Jeanneret 2007).
} 


\section{La « vue » comme étalon contesté}

À y regarder de près, la " vue » et ses chiffres paraissent se dissoudre dans une grande variété de formats et d'espaces médiatiques: des agrégats statistiques, des dispositifs d'observation des activités, des mots-clés, des bases de données, des ensembles de données de profils et de groupes d'utilisateurs. Dans les brevets, qui représentent à leur manière les relations entre toutes ces situations, la "vue » ne vaut que parce qu'elle permet de donner de la densité à des mots-clés et de les hiérarchiser. Cette vision myope masque cependant l'essentiel: l'importance de la "vue " tient à sa manière d'organiser des processus plus larges et de servir d'étalon dans les références à la "popularité " et à la "créativité ». Les chiffres de "vues " se font le signe de la circulation des tubes, en matérialisant l'objectif des musiciens que YouTube prétend accompagner, en témoignant de la puissance de YouTube dans le monde de la musique, ou encore en apparaissant comme l'unité de référence reliant les objectifs diversifiés des publicitaires.

\section{1 "Impact Metrics » : la vue ne tient qu'à un clic}

Dans toutes ces situations, la « vue » et ses chiffres se doivent d'être crédibles. La confiance dans sa capacité à indexer quelque chose sont la condition de possibilité de son efficacité : une mesure d'audience n'est pas qu'un « artefact (encore moins une manipulation) où seul compterait l'accord entre les parties ; pour pouvoir être utilisée, la mesure nécessite une forme de plausibilité » (Méadel, 2010, p. 211). C'est la condition pour qu'existe un marché, c'est-à-dire pour que des relations de confiance interpersonnelles deviennent des relations systématiques, organisées comme des transactions, sur le principe d'un bénéfice futur: les "vues" décomptées par YouTube permettent d'espérer des investissements publicitaires, elles promettent aux annonceurs qu'une vidéo ou qu'un mot-clé se rapprocheront d'un "intérêt» ou d'une disponibilité de l'internaute dans le futur, elles laissent espérer aux artistes qu'ils engrangeront un capital de notoriété et de visibilité convertible dans d'autres situations.

La « vue » prend donc une valeur fiduciaire, ce qui signifie qu'on considère qu'elle constitue une convention avec sa part de 
Vue vérifiées, écoute évacuée :

la valorisation publicitaire de la musique sur YouTube

croyance, un opérateur d'équivalence en même temps qu'un étalon qui offre sa part de légitimité à l'ensemble du système. Sur quoi ces attentes reposent-elles au fond? Tous les investissements - symboliques et financiers - dans la "vue » n'existent que parce qu'est reconnue l'idée qu'à un moment, le dispositif a enregistré quelque chose qui s'est traduit par l'incrémentation d'un compteur de vues, puis d'autres valeurs dans des bases de données. Si la vue tient comme forme de monnaie d'échange, c'est parce qu'elle est censée indexer quelque chose. Mais quoi ? II va de soi qu'une vue ici n'est pas un globe oculaire arraché à un visage d'internaute, un idéal vampirique dont semblent parfois se rapprocher les technologies d'eye-tracking.

Selon le texte Impact Metrics publié en 2016 par YouTube ${ }^{17}$, une " vue » correspondait alors seulement à un clic à n'importe quel endroit sur la barre de lecture, enregistré et décompté. Aussi, elle n'était pas liée à la durée continue et qualifiée de " visionnage » ni même de " consommation ». Elle faisait plutôt référence à l'enregistrement par la machine d'un contact probable, stocké et transmis par de multiples protocoles et reconstruit comme un regard ponctuel. Chaque clic sur la barre de lecture d'une vidéo, sans rechargement de la page, est alors compté comme une "vue »: une consultation d'un simple fragment de vidéo, sur le mode de l'aperçu, suffit à incrémenter le chiffre. "Vue » est alors le nom hyperbolique donné à ce point de contact minimal, caution de tous les réseaux logiques de traitement et d'inférences entre les « valeurs " et de toutes les transmutations entre mots, chiffres et symboles graphiques.

\subsection{La critique de l' " a-phorisme des vues ॥}

Étant donné le minimalisme du critère de comptage, la « vue » comme mesure d'audience a fait l'objet de mises à l'épreuve, même si elles n'ont pas nécessairement eu la portée d'une remise en cause intégrale. Ces mises à l'épreuve sont d'autant plus fortes que la réalisation des mesures d'audience est de moins en moins l'affaire des seuls publicitaires. De manière générale, les dispositifs médiatiques en ligne déstabilisent la figure du consommateur comme « téléspectateur quantifié, cette

17 YouTube. (2016). Impact Metrics. YouTube Creator Academy. Récupéré de https://creatoracademy.youtube.com/page/lesson/impact-metrics

[consulté le 11 janvier 2018 ; page retirée] 
commode fiction sur laquelle tout le monde pouvait s'entendre ", au profit de celle d'un internaute que les professionnels peinent parfois à caractériser dans son profil comme dans ses activités (Méadel, 2010, p. 242).

Les «vues" n'ont donc pas manqué de faire l'objet de critiques publiques, en particulier dans le monde de la musique, qui interroge leur consistance et les inférences qu'elles autorisent. Ironiquement, cette critique a pu s'appuyer sur les autres indicateurs proposés par YouTube : ainsi, les 64 millions de " vues » recensées en mars 2011 pour la chanson " Friday » de Rebecca Black n'ont pas empêché plus d'un million de dislikes (le pouce en bas), conduisant la critique musicale à défaire l'association entre "vue » et popularité pour la consacrer "vidéo YouTube la plus détestée de tous les temps ${ }^{18}$ ". Cet épisode médiatique attire l'attention sur les suites de ce qu'Yves Jeanneret et Valérie Patrin-Leclerc (2003) ont décrit à partir du cas de Loft Story: la capacité d'un programme à susciter une multiplicité de prises de position - soigneusement provoquées, dans ce cas précis - pour faire gonfler les chiffres d'audience en entretenant la confusion entre mesure d'audience, intérêt du public et « goût » ou satisfaction.

Ce programme et sa médiatisation représentent pour les auteurs

une sortie du système de la critique, dans lequel le succès dépend de discours promotionnels sur les productions culturelles, pour entrer dans celui de la mesure d'audience, travail purement quantitatif sur l'exposition au média [...]. "Faire de l'audience » est avec Loft Story une réalité ouvertement distincte de "plaire aux téléspectateurs » (paragr. 20).

Si rien n'indique que la chanson de Rebecca Black ait été publiée dans l'attente délibérée de réactions négatives ou polémiques, elle marque un épisode de prise de conscience de cette disjonction possible entre chiffres de "vue " et « popularité » au sens du plébiscite. À l'inverse, c'est bien cette relation a-phorique impliquée par les chiffres de "vues " que YouTube choisit de retraduire positivement (en l'hybridant avec

\footnotetext{
18 Matyszczyk, C. (2011, 29 mars). Rebecca Black passes Bieber as YouTube's most hated video. CNET. Récupéré de https://www.cnet.com/news/rebecca-black-passesbieber-as-youtubes-most-hated-videol
} 
Vue vérifiées, écoute évacuée :

la valorisation publicitaire de la musique sur YouTube

d'autres indicateurs qui peuvent sembler moins ambigus, comme les « pouces ») dans son vocabulaire de l'« intérêt ».

\section{3 « Vues véritables » et vues vérifiées : la certification du flou}

D'autres critiques plus fermes du statut de la " vue » ont concerné la prétention à enregistrer effectivement les activités des internautes. La presse a révélé qu'une vedette de la chanson comme Lady Gaga était parvenue à gonfler artificiellement les chiffres du compteur de vues, sans doute aux moyens de sociétés tierces spécialisées dans des techniques comme les leurres (Després-Lonnet, 2004), les incitations associées à des concours $^{19}$, voire les microrémunérations d'individus participant à des schémas pyramidaux ou à des " usines à clics " (Cardon et Casilli, 2015).

À défaut de pouvoir les empêcher, YouTube a reconnu l'existence de ce type de pratiques, pour ensuite mieux les décourager en critiquant leur efficacité du point de vue de l'efficacité publicitaire. L'entreprise a publié plusieurs communiqués à ce sujet, empruntant le vocabulaire du « contrôle qualité " et de l'audit financier pour rassurer les annonceurs $^{20}$. Elle s'est alors positionnée structurellement comme juge et partie, incarnant le média en même temps que la régie publicitaire, l'institut d'études et même le prestataire technique des systèmes de contrôle. Les agences extérieures qui voudraient revendiquer ce contrôle ${ }^{21}$ restent réduites à « capter » les données que YouTube propose via son API ${ }^{22}$ pour

\footnotetext{
${ }^{19}$ Ackerman Greenberg, D. (2007, 22 novembre). The Secret Strategies Behind Many "Viral" Videos. Récupéré de https://techcrunch.com/2007/11/22/the-secret-strategiesbehind-many-viral-videos/

20. Pferffenberger, P. (2014, 4 février.). Keeping YouTube Views Authentic. Google Online Security Blog. Récupéré de https://security.googleblog.com/2014/02/keepingyoutube-views-authentic.html

${ }^{21}$ C'est même YouTube qui recense et certifie ces chiffres, aux côtés d'une large gamme d'entreprises qui s'appuient sur les activités du site et des internautes et font donc partie de son "écosystème " économique (voir: YouTube Creator Services Directory. [s. d.]. Récupéré de https://servicesdirectory.withyoutube.com/ [consulté le 9 juillet 2018]

22 Pour Application programming interface (interface de programmation) : «Ensemble de routines standard facilitant le développement d'applications ou la manipulation d'opérations sur une plateforme [...]. Une API est constituée de l'infrastructure technique permettant l'accès - l'interface à proprement parler, les serveurs et protocoles d'authentification pour accéder aux données - et de la documentation, soit
} 
les interpréter à leur manière, par exemple en les croisant avec les données d'autres sites de publication. YouTube continue par ailleurs d'insister sur la fiabilité et la certification de sa mesure d'audience dans les pages à destination des annonceurs (YouTube For Advertisers) et à travers les pages de création de campagnes (le ratio argent/vues sur AdWords naturalise la vue comme indice de l'efficacité postulée de l'exposition sur YouTube).

Au-delà des discours de réassurance, l'entreprise a fait évoluer ses pratiques pour détecter les manipulations et rassurer ses différents publics. Pour les vidéos proposés par des annonceurs (dont les vidéos hébergées sur YouTube et transformées en message publicitaire par l'achat d'espace), la formule récurrente TrueView ("vue véritable ») est une manière de reconnaître le problème et de prétendre y répondre par une solution technique. En particulier, avec le format TrueView In Stream, l'utilisateur, au bout de cinq secondes d'une publicité en pre-roll, est invité à cliquer pour " sauter la pub » (« skip ad ») et visionner directement la vidéo qu'il a choisie. L'annonceur paye seulement si celui-ci continue de regarder le spot après ces cinq secondes, le regarde au moins trente secondes, jusqu'à la fin de la vidéo (selon ce qui est le plus court) ou encore s'il clique sur des " cartes " ou d'autres éléments du message. YouTube met donc en œuvre une sorte d'indice de " non-zapping », s'efforçant d'ancrer la "vue " dans une meilleure approximation des usages - dans l'hypothèse où ces règles ne seraient pas détournées à leur tour.

Pour le reste des internautes, et en particulier ses publics d'éditeurs ou de gestionnaires de chaînes et de producteurs de vidéos, YouTube a enrichi YouTube Analytics en ajoutant au nombre de vue un indicateur de temps de visionnage (Watch Time), souvent réinterprété dans la langue publicitaire en " rétention ». Enfin, et surtout, ses explications sur la façon dont les vues sont comptées explicitent aujourd'hui une méthode en deux temps, qui consiste à faire tourner des méthodes d'analyse dès qu'une vidéo franchit les 300 vues. Nul ne sait sur quel critère exact se fonde cette démarche de vérification, mais deux hypothèses sont régulièrement soulevées par les spécialistes en marketing en ligne qui s'adonnent à des tests de rétro-

l'ensemble des textes qui expliquent comment sont structurées les données et comment y accéder ». (Goyet, 2017, Tome II, p. 4). 
Vue vérifiées, écoute évacuée :

la valorisation publicitaire de la musique sur YouTube

ingénierie : un ou plusieurs rechargements de la page à partir d'une même adresse IP pourraient bloquer l'incrémentation, ce qui signifie que la "vue » renverrait moins à un compte de visionnage qu'à un compte de visionnage par des internautes distincts, du moins passé un certain seuil ; et le critère du " clic » serait en réalité remplacé par un critère de temps de visionnage minimal (identifié de 5 secondes à 30 secondes, selon les sources). Bien qu'elle puisse paraître justifiée par la nécessité de prévenir les tentatives de trucages, on ne peut que s'étonner de l'écart entre l'importance de cette mesure d'audience dans les stratégies de construction de notoriété en ligne et dans des flux de rémunération (puisque les accords de reversement des revenus publicitaires par YouTube se font en partie au prorata du nombre de vues par vidéo ${ }^{23}$ ) et le défaut d'information sur les procédures exactes sur laquelle elle repose.

Dans la mesure où elle donne lieu à des inscriptions, la disponibilité aux publicités fait partie de ce qu'Arjun Appadurai (1986) nomme des marchandises "par métamorphose ": des choses "placées dans un état de marchandise " même si ce n'est pas leur usage initial - en l'occurrence, il n'est même pas certain que l'on puisse parler de "choses". Cette métamorphose passe par une ingénierie des inscriptions et des symboles qui présente de multiples points de discontinuité et de fragilité : existence même d'un référent au signe (comme dans le cas des trucages des "vues ») ; qualité de la relation du signe au référent (la possibilité de lier le décompte d'un clic ou d'un temps passé à la texture phénoménologique d'une présence ou d'une expérience) ; ou encore qualification du référent lui-même (comment identifier ce pour quoi vaut le clic ou le défilement d'une vidéo). C'est pourquoi la combinaison de la référence à la

\footnotetext{
23 II existe plusieurs modalités de rémunération des artistes musiciens ou interprètes sur YouTube. Du point de vue d'une étude des calculs de «monétisation » par les " vues » (ce qui exclut, par exemple, les placements de produit), les principales sont: un pourcentage des revenus publicitaires issus des vidéos d'une chaîne propriétaire ("Programme Partenaire »), payés via l'outil AdSense ; un pourcentage des revenus publicitaires liés à la vente de publicités sur des vidéos incluant des œuvres musicales; et un pourcentage des revenus issus des accords de licence et des abonnements liés au service YouTube Premium (formule de visionnage et d'écoute sans publicité). Dans ces deux derniers cas, les sommes sont collectées par les représentants des artistes (maisons de disques, éditeurs ou distributeurs de musique numérique) et partagés avec eux selon les termes des contrats qui les associent (voir : YouTube Creator Academy. (2017, 11 juillet). Générer des revenus sur YouTube avec votre musique. Récupéré de https://creatoracademy.youtube.com/page/lesson/artistmonetization).
} 
vue avec des indices des temps de visionnage (TrueView) et des mots-clés sert de garantie d'une activité humaine, sa description psychologique pouvant dès lors insister alternativement sur les dimensions phoriques (les "pouces » qui disent " J'aime »), sensorielles (la "vue »), cognitives (l' " intérêt »), ou affectives (les commentaires, le cas de Rebecca Black). Néanmoins, le déplacement de la valeur économique d'une unité de clic (une "vue ») vers un décompte plus précis du temps (True View), comme la formulation de la continuité entre activité de l'internaute, clics et "intérêt ${ }^{24}$ ", ne change pas la logique fondamentale. YouTube prétend proposer la meilleure approximation possible de ce que les internautes font, désirent et ressentent, en passant par toute l'épaisseur et l'ambiguïté que suppose la manipulation des symboles et leur articulation vis-àvis du tissu de l'expérience.

\section{La musique évacuée}

Les questions de la définition de l'expérience, de l'attention, du temps passé ou pris sont au centre d'une multiplicité de discours qui associent le marché de la publicité en ligne à une " économie de l'attention ". La formule fait notamment suite aux travaux de Herbert Simon (1971) sur le management des ressources humaines dans les organisations, puis de Georg Franck (cité dans Citton, 2014a). Les discussions qui ont accompagné la diffusion de ces théories oscillent entre l'identification d'une écologie de l'expérience considérée dans sa labilité et dans son environnement (Citton 2014a) et l'analyse de la performativité des discours et des dispositifs économiques et

\footnotetext{
24 « Nous pensons que nous pouvons rendre la publicité en ligne encore plus pertinente et utile en utilisant des informations supplémentaires sur les sites web que les gens visitent. Aujourd'hui, nous lançons une publicité "basée sur les intérêts" sous forme de bêta test sur nos sites partenaires et sur YouTube. Ces publicités associeront des catégories d'intérêt - par exemple, le sport, le jardinage, les voitures, les animaux domestiques - à votre navigateur, en fonction des types de sites que vous visitez et des pages que vous consultez. Nous pouvons ensuite utiliser ces catégories d'intérêt pour vous présenter un texte plus pertinent et afficher des annonces ». Traduction libre de : "We think we can make online advertising even more relevant and useful by using additional information about the websites people visit. Today we are launching "interest-based" advertising as a beta test on our partner sites and on YouTube. These ads will associate categories of interest - say sports, gardening, cars, pets - with your browser, based on the types of sites you visit and the pages you view. We may then use those interest categories to show you more relevant text and display ads " (Wojcicki, S. (2009, 3 novembre). Making ads more interesting. Récupéré de https ://googleblog.blogspot.com/2009/03/making-ads-more-interesting.html).
} 
Vue vérifiées, écoute évacuée :

la valorisation publicitaire de la musique sur YouTube

gestionnaires. Ces approches avancent l'idée selon laquelle l' " attention " n'est pas une donnée immédiate, mais plutôt une façon dont ces discours et ces dispositifs choisissent de requalifier le cours des dispositions, des expériences ou des activités pour en faire des marchandises.

\subsection{Au-delà de l'attention : le temps comme marchandise par excellence}

D'un côté, ces théories décrivent comment l'attention humaine, c'est-à-dire la capacité à se rendre sensible à un milieu ou des objets de manière plus ou moins orientée, est altérée par les dispositifs médiatiques et publicitaires. De l'autre, elles se proposent de décrire l'attention comme une fiction ou une abstraction rendue crédible par une multiplicité de dispositifs, dans le cadre de la construction d'une modernité de l'écoute et du regard. À partir du XIXème siècle principalement, une multitude de dispositifs sont conçus qui nient l'irréductible différence entre le flux de la conscience (l'expérience qualitative des pensées, des sensations, de la durée, qu'elles soient entremêlées ou non) et l'objectivité du temps associé aux enregistrements, calculé par les machines et articulé à une activité cadrée et orientée de l'individu comme "récepteur " : c'est le cas de la télévision dans le domaine du visible (Crary, 2016) comme du phonographe, qui déplace dans l'espace du foyer la technique sensorielle de l'écoute orientée et attentive (Sterne, 2015).

De manière plus générale, le concept d'attention s'est en partie fondé sur la rétrojection des procédures de la médecine expérimentale, et en particulier de la psychologie expérimentale, dans une figure positiviste de la subjectivité : le psychologue Gustav Fechner est présenté comme une figure pivot dans la démarche qui a consisté, à partir de la seconde moitié du $\mathrm{XIXème}$ siècle, à réinventer la vision comme un phénomène "psychophysique », qui devient mesurable par des chiffres, indépendamment de la singularité des choses vues, des personnes et de leurs environnements (Crary, 2016). Cette figure du sujet attentif sert ensuite de soutien symbolique aux efforts pour mesurer et rationnaliser les expériences sensibles. Sous l'impulsion d'un souci généralisé de quantifier le réel, des discours scientifiques et des dispositifs instituent une figure de l'observateur moderne rendu autonome de son environnement culturel-naturel, et ses sensations auditives, visuelles et tactiles 
sont différenciées et susceptibles de maîtrise.

Le compte des vues par YouTube présuppose l'existence d'une telle formalisation de la perception et de cette abstraction du temps. Ces catégories idéalistes sont nécessaires pour pouvoir ramener la diversité des expériences des internautes, des spectateurs et des auditeurs à un même chiffre et pour donner à ce chiffre une valeur publicitaire et financière. II faut remarquer que le modèle du temps devenu marchandise ne saurait se réduire au résultat de la modernité technique, pas plus que de l'assomption d'une économie publicitaire ou d'une " économie de l'attention ». En effet, une telle rationalisation du temps est au centre du contrat de travail capitaliste : comme le remarquait Karl Marx (2009), la force de travail s'y trouve réindexée sur un temps de travail et c'est dans cette conversion qu'elle devient marchandise. Parce qu'il est au fondement du mode de production et d'échange qui distingue une économie capitaliste, ce temps-marchandise soutient donc dans ce régime économique la valeur de n'importe quel produit. II est justement ce qui fait que différentes activités humaines peuvent se voir reconsidérées comme des produits: "ce qui rend la marchandise échangeable, c'est l'unité de temps de travail social abstrait, car à travers la réduction à l'unité, on fait abstraction de la valeur d'usage, et des besoins »(Adorno, 2018, s. p.). Le travail industriel et salarié fait ainsi du temps lui-même l'un des fondements de la valeur d'échange.

Dans la réflexion sur l'extension de ce paradigme au-delà du périmètre du travail industriel, et en particulier vers le loisir et les pratiques culturelles, on peut se demander dans quelle mesure la pensée de la musique aura contribué à forger une norme du temps " bien investi ». La tradition musicale dite "classique », à travers des dispositifs comme la mesure ou le métronome, a pu accompagner cette temporalité abstraite, même si la musique a aussi souvent servi de métaphore à tout ce qui échappe à la mesure du temps ("flux», "immersion», etc.). À partir du travail industriel et des instruments de mesure, le temps peut servir non seulement de repère social mais aussi de valeur économique omniprésente, traduisant les autres valeurs ${ }^{25}$.

\footnotetext{
25 « La valeur capitaliste ne peut se former que si les unités de temps abstrait existent mais ces unités de temps abstrait doivent avoir une objectivité sociale - c'est-à-dire que chacun des acteurs ou actrices engagés sur le marché doit les reconnaître et les utiliser - pour que cette valorisation rétroactive sur le marché se produise et pour que
} 
Vue vérifiées, écoute évacuée :

la valorisation publicitaire de la musique sur YouTube

C'est cette valeur symbolique et économique fondamentale implicite dans la référence à la « vue » qu'est venue révéler la requalification du fondement de la « vue » de simple " clic » aux "vues véritables" et autres «vues" vérifiées (par la présomption d'une durée investie). Ces variantes construisent l'image d'un " temps choisi » d'un internaute investi, disponible, impliqué, isolé de son environnement, situé dans un pur face à face avec la vidéo ou de morceau de musique en train de se lire, maître de sa conscience jusqu'à en faire coïncider les mouvements intensifs avec ceux d'un calcul homogène du temps. Dans ce paradigme, non seulement la durée spécifique à l'expérience d'une œuvre elle-même spécifique se trouve réglée sur le temps mesurable d'un enregistrement, mais les diverses expériences potentielles d'une œuvre par différents individus deviennent aussitôt commensurables entre elles. Cette fiction d'écoute ou de consommation objective et égale contraste autant avec les usages des internautes - qui ne sont pas nécessairement présents ou attentifs quand la lecture " tourne » - que les expériences d'auditeurs - une écoute «coup de cœur ", ou encore le "choc» du spectateur benjaminien (Benjamin, 2000) peuvent suspendre l'épreuve de la durée - ou des esthètes - la fameuse "écoute structurelle " d'Adorno (1994), qui ne cesse d'éprouver les rapports entre fragments et totalité, creuse une temporalité tangentielle dans le temps de l'exécution de l'œuvre et de sa « reproduction » par la machine.

\subsection{La spécification et la purification du sensible}

Compter des clics comme des "vues" pour les afficher sur les pages, certifier auprès des annonceurs des «vues véritables ", car excédant un temps de zapping (True View), ou encore décompter du « temps de visionnage " pour renseigner les éditeurs de chaînes, ce n'est donc pas seulement chercher à rendre compte de ce que les internautes font en lançant des vidéos : c'est d'entrée de jeu promettre une attention " purifiée ", isolée de toute interférence, réglée sur un objet choisi. Pour lier la musique à cet idéal, il suffit d'étendre ce modèle quantitatif de la perception en ajoutant l'indistinction entre les facultés ou les techniques sensorielles: si l'activité du regard est réduite

la loi de la valeur s'y impose. Cette forme de temps abstrait doit englober tout le champ social pour participer à la formation de la valeur, synchroniser et coordonner la production, la circulation, et ainsi de suite » (Martineau et St-Hilaire, 2017). 
théoriquement à une activité mesurable et dénombrable, à un pur « visionnage », alors le fait d'entendre peut bien valoir une vue.

Pour Jonathan Crary (2016), la formalisation de la vue par Fechner exprimait «la proximité, voire la coïncidence, de l'expérience sensorielle et d'un terrain économique et social dominé par les valeurs d'échange »(p.211), dans lequel les dispositifs de mesure de la vision « permettent de gouverner le sujet percevant, de le prévoir, de le rendre productif et, pardessus tout, de le mettre au diapason avec les autres domaines de la rationalisation » (p. 227). L'ouïe a connu le même type de "purification" scientifique que la vision. Avant même la modélisation psycho-physique, l'idée d'une perception auditive adéquate à l'œuvre et valorisée comme telle était déjà inscrite dans la tradition occidentale des discours sur la musique, que ce soit dans la dominance du discours sur la musique comme rhétorique des affects au XVIII ème siècle, dans la valorisation progressive d'une attention claire et distincte dans laquelle la raison guide les sensations au XIXème siècle, ou dans le discours sur l'écoute esthésique au XXème siècle (Kaltenecker, 2011).

La manière dont les savants et les ingénieurs ont imaginé à leur tour l'écoute comme phénomène transindividuel, objectivable et mesurable par des chiffres résonne donc avec cet idéal de rationalité de l'écoute spécifiquement musicale et " classique ». L'invention du son comme dimension transversale à la musique et à la parole, opposé au bruit, répond ainsi à l'exclusion de "tout contenu précis » dans la vision, qui avait déjà permis, de son côté, une "homogénéisation " avec les autres sensations (Crary, 2016). C'est bien en raison d'une telle purification qu'au tout début du XXème siècle, Georg Simmel (2003) pouvait poser l'hypothèse d'un goût esthétique explicable par un facteur quantitatif d'" excitation » physiologique.

Le travail de YouTube sur la mesure d'audience s'inscrit dans une telle dynamique historique. En même temps, ces remarques éclairent les définitions et les hiérarchies implicites des facultés sensorielles qui nourrissent les pratiques économiques. C'est parce que la vision s'est révélée historiquement objectivable et administrable, notamment à travers les appareils de visionnage et de mesure d'audience, que les instruments de sa gestion et de son contrôle peuvent être reconduits et adaptés d'un dispositif médiatique à l'autre. D'un autre côté, la réussite de l'entreprise 
Vue vérifiées, écoute évacuée :

la valorisation publicitaire de la musique sur YouTube

de quantification scientifique et d'homogénéisation imaginaire des facultés sensorielles leur a fait perdre une part de leur spécificité dans l'imaginaire social, et c'est ce qui autorise aujourd'hui à valoriser les relations à la musique médiatisée comme des "vues » ou du "temps passé » sur le fond d'une même idée d'un consommateur de médias disponible et intéressé.

Du point de vue de l'affichage des publicités sur YouTube, la différence entre regard et audition importe peu : un morceau de musique dans le lecteur-média (player) se lance comme n'importe quelle vidéo, et peu importe qu'il soit visionné ou apprécié selon des techniques sensorielles distinctes. Comme nous l'avons vu, l'appariement entre publicité et enregistrements est surtout sémantique, il passe par les mots-clés, dans une logique qui motive la formule omniprésente de " contenu ». Les catégories des " audiences d'affinité » vendues aux annonceurs (« amateur de pop ») se situent sur le plan des pratiques de consommation, des genres musicaux comme réalités "transmédiatiques » et des formats de vidéos (clips, cours de musique) : elles ne sont jamais reliées à des dispositions issues d'une culture particulière du son ou de l'écoute comme dimensions distinctes. Cette imprécision ne poserait problème que dans le scénario où YouTube voudrait vendre aux annonceurs de l'écoute plutôt que du visionnage.

\subsection{La valorisation de la circulation}

Comment, dans ce contexte, établir ce qui est perçu et approprié par les internautes et la part du son et de l'écoute dans leurs pratiques ? Si la "vue " est une unité de consommation qui déduit du temps machine un temps investi par l'internaute, comment savoir si ce temps doit être virtuellement interprété comme un temps pour la musique ou pour la vidéo?

Cette question importe pour au moins deux raisons : d'une part, contrairement à la thèse d'une indifférence structurelle des " plateformes " aux pratiques culturelles, YouTube a bâti une partie de sa stratégie sur des formes d'éditorialisation spécifiques de la musique ${ }^{26}$. Par ailleurs, sa centralité dans les

${ }^{26}$ YouTube a régulièrement saisi les occasions que représentait le fait d'étoffer son travail éditorial sur la musique. L'entreprise construit de nombreuses médiations pour attirer les musiciens et leur vanter un modèle de promotion et de financement « direct- 


\section{Guillaume HEUGUET}

pratiques de la musique en ligne dépend de l'accord des maisons de disques et d'autres ayants droit pour l'exploitation de leurs catalogues, cet accord reposant lui-même sur la capacité de YouTube à convaincre qu'elle rémunère cet usage à hauteur de ce qu'ils lui rapportent (ce sont les controverses dites de l'« écart de valeur " ou "value gap ») ou des pratiques qu'elle autorise.

En 2014, l'entreprise a répondu à la question de la spécificité de la musique dans le compte des "vues ». Les "titres » déposés par des chaînes d'un "artiste officiel »" par un distributeur de musique numérique pour être administrés via l'outil de contrôle des droits Content ID font l'objet d'un mode de calcul statistique particulier: le total des vues d'un titre correspond au nombre de vues des vidéos utilisant l'intégralité ou seulement une partie du morceau, y compris les vidéos officielles, les vidéos en direct, les contenus créés par des fans et les publicités ${ }^{28}$. Toute consultation d'une vidéo où figure un morceau (déclaré comme composition ou comme enregistrement par les ayants droit ${ }^{29}$ ) est donc comptée comme

to-fan ». Elle a organisé des concours, s'est approprié l'aura d'artistes et de scènes émergentes et a cherché à se rendre responsable de "tubes YouTube ". En plus d'établir des partenariats avec les principales majors, l'entreprise a développé une éditorialisation renforcée de la musique en page d'accueil à partir de 2015. Elle a notamment inauguré le format des playlists musicales orientées sur des contextes d'usage et des " humeurs " et expérimenté des systèmes d'écoute en continu fondé sur les recommandations (YouTube Mix). Enfin, cette stratégie éditoriale a très vite été associée à une offre spécifique de streaming musical payant, YouTube Music, sur ordinateur et sur téléphone mobile.

27 Un compte "Official Artist » pour un artiste musicien ou interprète passe par une procédure différente qui requiert d'abord d'avoir le "Topic Channel » généré à son nom par YouTube selon des critères comme le nombre de vidéos, le nombre de vues, et la " qualité » associée à un nom d'artiste. Il est ensuite nécessaire de créer une chaîne à son nom d'artiste (interprète ou musicien) et de faire une demande de qualification par e-mail auprès de YouTube (voir: YouTube (s. d.). Official Artist Channel Playbook. Répéré à https://services.google.com/fh/files/helpcenter/oac playbook.pdf). Pour devenir éligible au "Partner program», qui autorise la " monétisation » par la publicité, la chaîne peut notamment justifier de 4000 heures de visionnage dans les 12 derniers mois et d'un minimum de 1000 abonnés.

28 YouTube Music Charts. Disponible à l'adresse : https://charts.youtube.com/?hl=fr. [Consulté 4 octobre 2018]. Ce texte ne figure plus sur cette page à la date de révision de cet article.

29 Composition et enregistrement correspondent aux deux statuts distincts de l'« œuvre musicale », tels qu'établis par le droit et par les pratiques des sociétés de gestion (voir : Aide YouTube (s. d.). Contenus éligibles dans Content ID. Récupéré de https://support.google.com/youtube/answer/2605065?hl=fr [consulté 9 juillet 2018]). YouTube a conclu ses premiers accords avec un grand nombre de sociétés de gestion des droits patrimoniaux en 2012 (Moody, E. (2012, 6 mai). Sing it! YouTube opens the door for more songwriters, publishers and content creators. YouTube Official Blog. 
Vue vérifiées, écoute évacuée :

la valorisation publicitaire de la musique sur YouTube

une " vue " pour l'artiste et le morceau en question, quelle que soit la durée du morceau dans la vidéo ou l'usage qui en est fait : c'est la "vue ${ }^{30}$ de la vidéo dans laquelle celle-ci se trouve incorporée qui s'avère déterminante.

Ces vues ne seront donc pas nécessairement liées à un type de vidéos, du type archive musicale ou " clip officiel », ancrant a minima la musique dans une culture de l'écoute ou des médias musicaux. Avec ce principe, un morceau de musique pourrait passer inaperçu et déplaire dans une vidéo qui connaîtrait un grand succès pour d'autres raisons, sans que cela affecte le compte de ses "vues ॥. Il pourrait aussi connaître le succès rien qu'en servant de support à des vidéos parodiques, ou de générique.

II est certes difficile de pondérer l'importance relative de ce critère de calcul par rapport à d'autres facteurs qui informent la remontée dans les systèmes de recommandation, donc l'exposition et l'audience cumulée ${ }^{31}$. Ce qui importe est néanmoins la façon dont, sous une référence lexicale apparemment consensuelle comme la "vue », un mode de calcul peut modifier les valeurs qui font la notoriété des œuvres et à partir desquelles les artistes peuvent revendiquer des revenus.

La détermination de la valeur des œuvres musicales par des chiffres de ventes, des diffusions radiophoniques et locales, elles-mêmes dépendantes de procédures de calcul contestées et relativement évolutives ${ }^{32}$, s'ancrait surtout dans l'idée d'un goût lié à des « pratiques de consommation », même indirectes ou reconstruites ${ }^{33}$. Sur YouTube, bien qu'il soit en pratique

Récupéré de https://youtube.googleblog.com/2012/06/sing-it-youtube-opens-door-formore.html).

${ }^{30}$ Donc le clic assorti d'une durée minimale de visionnage inconnue, voire plus haut.

31 Les titres de majors du disque, bénéficiant par ailleurs de "clips officiels » et d'autres ressources promotionnelles (gestion de chaînes, référencement, relations presse, etc.), occupent l'écrasante majorité du "YouTube Music Chart » depuis son lancement. Une étude systématique de ce palmarès permettrait d'identifier les titres qui auraient pu bénéficier d'un effet indirect de diffusion par les vidéos de reprise.

32 On peut penser aux polémiques récurrentes sur les modes d'administration des droits et de redistribution retenus par la SACEM, ainsi qu'à ses efforts pour se doter de nouveaux outils de traçage.

33 L'ancrage dans la référence aux «pratiques de consommation » tendant nécessairement à mettre de côté la part potentielle des premières circulations, dont les campagnes de relations publiques, y compris intermédiatiques (campagnes de presse et de publicité) dans leur préformation: on ne "choisit » ou on ne paye que pour ce qui fait déjà partie de l'espace du marché. 
difficile de distinguer les choses, la circulation interne au média est devenue un critère potentiellement déterminant. Ce critère de circulation est en cohérence avec les axes retenus dans le système de recommandation qui favorise tendanciellement, outre l'inscription des vidéos dans des " chaînes YouTube » aux publications régulières, des phénomènes comme la vélocité initiale des vidéos, l'intersection entre thématiques sociales et culturelles (Rieder, Matamoros-Fernández Coromina, 2018) ou encore les formes et opinions polarisantes ${ }^{34}$.

Ce mécanisme fonctionne bien sûr comme une incitation aux ayants droit à autoriser les réappropriations de leurs morceaux, afin de profiter de l'exposition de ces palmarès, voire à s'arranger pour les encourager. Des entreprises y travaillent déjà, comme c'est le cas de Dance On, lancée avec l'aide de la chanteuse Madonna en 2011 pour initier des vidéos de danse pseudospontanées dans le cadre de la promotion de singles, en espérant des effets boule de neige. Si l'on suit cette logique, peu importe la place qui est réservée au son et à la forme musicale dans l'expérience qu'un internaute en fait dans une vidéo: la capacité de ce morceau à susciter une reprise - par exemple parce qu'il est facile à monter avec des images, ou parce qu'il a un fort pouvoir d'évocation - est structurellement déterminante.

Tendanciellement, n'importe quel morceau de musique repris dans de nombreuses vidéos à succès est susceptible de rivaliser avec un morceau de musique qui serait privé de telles reprises. Cette même logique du devenir-meme de la musique, qui consiste à miser sur l'articulation entre un morceau et la contagion d'une image, même parodique, pour accélérer sa circulation, domine désormais le fonctionnement de l'application à succès Tik Tok (ex-Byte Dance).

\section{Conclusion}

C'est donc un édifice fragile qui soutient les mesures d'audience des vidéos et en particulier celles de cette partie des vidéos qui compte comme "musique » sur le site. Le décompte du succès des vidéos musicales comme "vues " n'est pas un abus de langage témoignant du primat du visuel sur le son, ou

${ }^{34}$ Tufecki, Z. (2018m 16 janvier). It's the (democray-poisoning) Golden Age of free speech. Wired. Récupéré de https://www.wired.com/story/free-speech-issue-techturmoil-new-censorship/ 
Vue vérifiées, écoute évacuée :

la valorisation publicitaire de la musique sur YouTube

l'indice d'une indifférence aux " contenus ", dont nous avons montré au contraire à quel point ils étaient sans cesse sémantisés, qualifiés, retraduits. II signale que sur YouTube, la musique prend sa valeur sociale et économique sans qu'elle ait besoin d'être saisie séparément de ses associations visuelles et thématiques, appréciée, ou même tout simplement entendue. La musique est hiérarchisée à partir de l'attractivité des vidéos dont elle participe. Alors que l'entreprise insiste à l'occasion sur son souci de la valeur culturelle de la musique et de la rémunérer à hauteur de cette valeur (auprès des ayants droit) ou s'appuie sur des catégories comme l' "intérêt " thématique pour tel ou tel genre (pour inciter les annonceurs à acheter des publicités), le destin de la musique y dépend au moins en partie de sa capacité à œuvrer pour d'autres causes que la sienne.

\section{Références}

Adorno, T. W. (1994). Introduction à la sociologie de la musique. Genève, Suisse : Contrechamps Éditions.

Adorno, T. W. (2018). Sur Marx et les concepts de base de la théorie sociologique. Web-revue des industries culturelles. Récupéré de http://industrie-culturelle.fr/industrieculturelle/sur-marx-et-les-concepts-de-base-de-la-theoriesociologique-theodor-w-adorno/

Appadurai, A. (1986). The social life of things. Cambridge, Royame-Uni : Cambridge University Press.

Benjamin, W. (2000/1935). L'œuvre d'art à l'ère de la reproductibilité technique. Dans CEuvres. Tome III. Paris, France : Gallimard.

Beuscart, J. S. (2006). La construction du marché de la musique en ligne: L'insertion économique et juridique des innovations de diffusion musicale en France (Thèse de doctorat). ENS Cachan.

Bouchard, J., Candel, É., Cardy, H. et Gomez-Mejia, G. (2015). La médiatisation de l'évaluation/Evaluation in the Media. Berne, Suisse : Peter Lang AG.

Bullich, V. (2015). Régulation des pratiques amateurs et accompagnement de la professionnalisation : la stratégie de 
YouTube dans la course aux contenus exclusifs. Les Enjeux de l'Information et de la Communication, (16), 27-42.

Bullich, V. (2018). La "plateformisation » de la formation. Distances et médiations des savoirs. Distance and Mediation of Knowledge, (21). https://doi.org/10.4000/dms.2096

Callon, M., Licoppe, C. et Muniesa, F. (2003). Présentation. Réseaux, (122), 9-12.

Callon, M., et Muniesa, F. (2003). Les marchés économiques comme dispositifs collectifs de calcul. Réseaux, (122), 189-233.

Cardon, D. et Casilli, A. A. (2015). Qu'est-ce que le digital labor?. Paris, France : INA.

Citton, Y. (2014a). Pour une écologie de l'attention. Paris, France : Seuil.

Citton, Y. (2014b). L'économie de l'attention. Paris, France : La Découverte.

Crary, J. (2016). Techniques de l'observateur: vision et modernité au XIXe siècle. Belleveaux, France: Éditions Dehors.

Després-Lonnet, M. (2004). Écrits d'écran : le fatras sémiotique. Communication \& langages, (142), 33-42. https://doi.org/10.3406/colan.2004.3301

Eriksson, M. (2019). In Pursuit of Musical Identifications: YouTube Content ID and the Politics of Audio Fingerprint Technologies (Thèse de doctorat). Umea University.

Goyet, S. (2017). De briques et de blocs. La fonction éditoriale des interfaces de programmation (API) web: entre science combinatoire et industrie du texte (Thèse de doctorat). Celsa Paris-Sorbonne.

Gras, S.-E. (2014). L'écoute en ligne : figures du sujet écoutant et mutations des espaces musicaux sur Internet (Thèse de doctorat). Paris-Sorbonne.

Heuguet, G. (2018). Métamorphoses de la musique et capitalisme médiatique. Au prisme de YouTube (2005-2018) (Thèse de doctorat). Sorbonne université. 
Vue vérifiées, écoute évacuée :

la valorisation publicitaire de la musique sur YouTube

Heuguet, G. (2019). Vers une micropolitique des formats. Revue d'anthropologie des connaissances, 13(3), 817-848.

Hillrichs, R. (2005). Poetics of Early YouTube: Production, Performance, Success (Thèse de doctorat). Universitäts-und Landesbibliothek Bonn.

Jeanneret, Y. (2007). Y a-t-il (vraiment) des technologies de l'information?. Villeneuve d'Ascq, France, Pays: Presses Universitaires du Septentrion.

Jeanneret, Y. (2014). Critique de la trivialité : Les médiations de la communication, enjeu de pouvoir. Le Havre, France: Éditions Non Standard.

Jeanneret, Y. (2019). La fabrique de la trace (vol. 2). Londres, Royaume-Uni : ISTE Group.

Jeanneret, Y. et Patrin-Leclère, V. (2003). Loft story 1 ou la critique prise au piège de l'audience. Hermès, La Revue, (37), 143-154.

Kaltenecker, M. (2011). L'oreille divisée: les discours sur l'écoute musicale aux XVIII et $X I X^{e}$ siècles. Les Douches, France : MF.

Martineau, J. et St-Hilaire, C. (2017). L'ère du temps : modernité capitaliste et aliénation temporelle. Montréal, Canada: Lux éditeur.

Marx, K. (2009). Le Capital I. Montreuil, France : Le temps des cerises.

Mclntosh, H. (2016). Vevo and the Business of Online Music Video Distribution. Popular Music and Society, 39(5), 487-500.

Méadel, C. (2010). Quantifier le public: histoire des mesures d'audience de la radio et de la télévision. Paris, France: Economica.

Mœglin, P. (2015). Pour une économie politique de la création. De la trivialité à la créativité. Communication \& langages, (185), 49-66.

Mulligan, M. (2015). Awakening: The music industry in the digital age. Hereford, Royaume-Uni : MIDiA Research. 
Muniesa, F., Millo, Y. et Callon, M. (2007). An Introduction to Market Devices. The Sociological Review, 55(2_suppl), 1-12. https://doi.org/10.1111/j.1467-954X.2007.00727.x

Perticoz, L. et Matthews, J. (2012). L'industrie musicale à l'aube du XXIème siècle. Approches critiques. Paris, France: L'Harmattan.

Polanyi, K. (2011). La Subsistance de l'homme: la place de l'économie dans l'histoire et la société. Paris, France: Flammarion.

Rebillard, F. et Smyranios, N. (2019). Quelle « plateformisation » de l'information? Collusion socioéconomique et dilution éditoriale entre les entreprises médiatiques et les infomédiaires de l'Internet. tic\&société, 13(1-2), 247-293.

Rieder, B., Matamoros-Fernández, A. et Coromina, Ò. (2018). From ranking algorithms to "ranking cultures" Investigating the modulation of visibility in YouTube search results. Convergence, 24(1), 50-68.

Simmel, G. (2003). Le cadre et autres essais. Paris, France : Gallimard.

Simon, H. A. (1971). Designing organizations for an informationrich world. M. Greenberger (dir.), Computers, communications, and the public interest (p. 37-72). Baltimore, Maryland : Johns Hopkins University Press.

Souchier, E. et Jeanneret, Y. (2009). Socio-sémiotique des médias. Dans D. Ablali et D. Ducard (dir.), Vocabulaire des études sémiotiques et sémiologiques (p. 145-150). Paris, France : Presses universitaires de Franche-Comté.

Sterne, J. (2015). Une histoire de la modernité sonore. Paris, France: La Découverte/Philharmonie de Paris-Cité de la musique.

Wade Morris, J. (2015). Selling digital music, formatting culture. Oakland, CA : University of California Press. 